\title{
La relación música-lengua materna en los principios \\ metodológicos de Edgar Willems y Shinichi Suzuki
}

\author{
A relação \\ música- língua \\ mãe nos princípios \\ metodológicos de \\ Edgar Willems e \\ Shinichi Suzuki
}

Music-

Mother tongue

Relationship in the

Methodological

Principles of

Edgar Willems and

Shinichi Suzuki

Narciso José López García* https://orcid.org/0000-0002-5665-5263

María del Valle De Moya Martínez** https://orcid.org/0000-0003-4701-4963

Raquel Bravo Marín*** http://orcid.org/0000-0001-5809-7739

Para citar este artículo

López García, N., Moya Martínez, M. y Bravo Marín, R. (2021). La relación música-lengua materna en los principios metodológicos de Edgar Willems y Shinichi Suzuki. Folios, (54).

https://doi.org/ 10.17227/folios.54-11512

* Doctor en Didácticas Específicas-Educación Musical de la Universidad de Málaga, España. Profesor Asociado del Departamento de Didáctica de la Expresión Musical de la Facultad de Educación, Universidad Castilla-La Mancha, Albacete, España.

Correo: narcisojose.lopez@uclm.es

* Titulada Superior en Musicología y en Lenguaje Musical del Real Conservatorio Superior de Música de Madrid, España y Profesional de Piano y Superior de Pedagogía Musical del Conservatorio Superior de Música, Madrid, España. Profesora Titular de Didáctica de la Expresión Musical de la Facultad de Educación, Universidad Castilla-La Mancha, Albacete, España

Correo: mariavallede.moya@uclm.es

*** Máster en Humanidades, Cultura y Sociedad de la Universidad Castilla-La Mancha, España. Profesora Asociada del Departamento de Didáctica de la Expresión Musical de la Facultad de Educación, Universidad de Castilla-La Mancha, Albacete, España.

Correo: raquel.bravo@uclm.es 


\title{
Resumen
}

La música, el lenguaje y sus relaciones como procesos de comunicación han sido objeto de numerosos estudios; lingüistas, musicólogos y pedagogos musicales de prestigio internacional se han dedicado a investigar las conexiones entre ambas disciplinas, sus paralelismos y sus divergencias. Este artículo de investigación se ha centrado en recoger las ideas de dos de las grandes figuras de la pedagogía musical, Edgar Willems y Shinichi Suzuki, y en exponer sus teorías relacionadas con la importancia del aprendizaje de la música y su desarrollo ligado al aprendizaje y desarrollo de la lengua materna. Para ello, se ha llevado a cabo una búsqueda, selección y análisis de información y documentación existente sobre este asunto con la finalidad de recoger, poner en valor, divulgar y clarificar todos aquellos aspectos presentes en ambas pedagogías musicales relacionados como la interrelación entre el aprendizaje musical y el aprendizaje de la lengua. Finalmente, se presentan las conclusiones generales derivadas de la investigación llevada a cabo en las que se pone de manifiesto dicha interrelación y se valoran las conexiones entre ambos sistemas de comunicación.

\section{Palabras clave}

educación musical; lengua materna; método Willems; método Suzuki

\begin{abstract}
Music, language and their relationships as communication processes have been the subject of numerous studies where linguists, musicologists and musical educators have been dedicated to investigate the connections between the two disciplines, their similarities and divergences. This research article has focused on collecting the ideas of two of the great figures of Musical Pedagogy, Edgar Willems and Shinichi Suzuki, where their theories on the importance of music learning and its development linked to learning and developing mother tongue are exposed. In methodological aspects this text has carried out a search, selection and analysis of existing information on this matter, collecting, valuing, disclosing and clarifying all those aspects present in both musical pedagogies. One of these features is the interrelationship between musical and language learning. Finally, the general conclusions arising from the investigation are carried out, showing the interrelationship and the connections between the two communication systems assessed.
\end{abstract}

$$
\begin{array}{r}
\text { Keywords } \\
\text { music education; mother tongue; Willems method; Suzuki method }
\end{array}
$$

\section{Resumo}

A música, a linguagem e suas relações como processos de comunicação têm sido objeto de inúmeros estudos. Linguistas, musicólogos e pedagogos de renome se dedicaram a investigar as vinculações entre as duas disciplinas, suas semelhanças e suas diferenças. Este artigo de pesquisa concentrou-se em coletar as ideias de duas das grandes figuras da Pedagogia Musical, Edgar Willems e Shinichi Suzuki, e em expor suas teorias relacionadas à importância da aprendizagem da música e seu relacionamento com a aprendizagem e o desenvolvimento da língua materna. Para isso, foi realizada uma pesquisa, seleção e análise da informação e documentação existentes sobre esse assunto, a fim de coletar, aperfeiçoar, divulgar e esclarecer todos os aspectos presentes nas pedagogias musicais relacionadas, como a inter-relação entre a aprendizagem musical e dos idiomas. Finalmente, são apresentadas as conclusões gerais derivadas das pesquisas realizadas, nas quais essa inter-relação manifesta-se, e são avaliadas e valorizadas as conexões entre os dois sistemas de comunicação.

\section{Palavras chave}

educação musical; língua materna; método Willems; método Suzuki 


\section{Introducción}

Estudios recientes indican que la música y el lenguaje comparten una red de procesamiento sintáctico común, abriendo camino a la posibilidad de que la activación de representaciones en el do minio musical facilite el procesamiento de representaciones paralelas en el dominio del lenguaje y viceversa.

En esta línea, un buen número de investigaciones se han centrado en buscar y analizar las coincidencias entre el aprendizaje musical y el aprendizaje del lenguaje en niños en edades tempranas. Para ello, se ha recurrido al terreno de la psicología del desarrollo y de la psicología de la música, lo que ha servido para encontrar tanto paralelismos como divergencias necesarias, entre otros aspectos, para comprobar la existencia de una escala evolutiva similar entre ambos aprendizajes, así como la presencia de características divergentes derivadas de los condicionantes propios y específicos de cada uno de estos dos sistemas significantes.

En las áreas de conocimiento directamente relacionadas con la música, expertos de la talla de Theodor Adorno o Enrico Fubini, entre otros, han dedicado gran parte de sus investigaciones a estudiar las conexiones entre música y lenguaje. Así, para Fubini (2004), las semejanzas radican en la organización sintáctica del material sonoro y, por tanto, en el aspecto discursivo de la música. Por su parte, Adorno $(1997,2000)$ señala que tanto lenguaje como música son sistemas significantes, característica que constituye uno de los fundamentos de su interconexión ya que, al igual que el lenguaje, la música comprende una lingüística y una sintaxis y, por tanto, es poseedora de la capacidad de transmitir un contenido.

Profundizando en este asunto, Eisner (2013) afirma que la música es semejante al lenguaje en cuanto sucesión temporal de sonidos articulados. Además, esta está formada por frases, periodos, puntuaciones, lo que pone de manifiesto la existencia de una organización sintáctica del material sonoro, condición sine qua non para que la música tenga un discurso coherente y no sea un grupo de sonidos amontonados.
Por otro lado, Igoa (2010) subraya que la música y el lenguaje tienen la capacidad de generar combinaciones infinitas a partir de un inventario finito de elementos en el que encontramos componentes comunes como:

- La percepción y la producción del sonido.

- La estructura combinatoria o la formación de secuencias complejas a partir de unidades básicas (notas y motivos-fonemas, sílabas y palabras).

- La expresión y la interpretación de intenciones y significados.

Asimismo, señala que la hipótesis de que música y lenguaje comparten diferentes recursos de procesamiento induce a pensar que la activación de representaciones en uno de los dominios puede producir efectos de priming ${ }^{1}$ en el dominio contrario, lo que facilitaría las representaciones paralelas (Igoa, 2010).

En este mismo sentido, Alonso, Suárez, Espinoza y Ramírez (2016) proponen una serie de aspectos asimilables entre música y lenguaje, de los que destacan los siguientes:

- Los relacionados con los patrones rítmicos, encargados de otorgar concordancia a la estructura silábica de una palabra.

- La estructura jerárquica que tanto música (armonía) como lenguaje (sintaxis) poseen.

- Las ideas, emociones o sentimientos que se quieren expresar necesitan de un conocimiento semántico que permita un hilo conductor.

- Las inflexiones del discurso hablado tienen una prosodia que se relaciona directamente con el mensaje que queremos comunicar, lo cual tiene su correspondencia con la melodía.

- Tanto la estructura general de la música como la del lenguaje contemplan un inicio,

1 El término priming hace referencia a la posibilidad real de que las experiencias previas ayudan a la realización de una tarea de la que, a priori, no tenemos experiencias con ella. 
un desarrollo y un final como organización básica de un discurso bien construido.

Palacios (2005), por su parte, nos recuerda que existe un buen número de investigaciones que se han centrado en estudiar las analogías entre la adquisición de destrezas musicales y destrezas lingüísticas y la transferencia de destrezas interdisciplinares.

Dos de las grandes figuras de la pedagogía musical, Edgar Willems y Shinichi Suzuki, centraron su trabajo en buscar estas analogías con la intención de localizar, si los hubiera, puntos de encuentro en el desarrollo de las capacidades lingüísticas y musicales de los niños, basando sus metodologías de aprendizaje musical en la relación entre la música y la lengua materna; el aprendizaje de la lengua materna es reflejo del aprendizaje musical en el sentido en que este, previo a la práctica, se basa en la escucha y en la imitación (Álamos y Pérez, 2015). Los niños están rodeados de sonidos del idioma de su madre desde antes de nacer. Si estuvieran en contacto con los mismos estímulos musicales podrían desarrollar una habilidad tan extraordinaria en la música como la que desarrollan en el lenguaje (Martí, 2016). Esta simbiosis será efectiva siempre que se den una observación, una audición, una imitación y una repetición adecuadas que, junto con la estimulación de los padres y de los profesores, se convierten en los pilares que sustentan sus teorías.

Así, de la lengua materna a la música solo hay un paso ya que, en ambos casos, nos hallamos en el mundo de los sonidos y, a partir de este principio, tanto Willems como Suzuki fijan un orden de desarrollo similar entre la capacidad lingüística y la capacidad musical (Bossuat, 2007).

Desde este mismo punto de vista, Saffran et ál. (1999), y Herrera y Lorenzo (2006) indican que aprender un lenguaje requiere aprender los elementos básicos necesarios para la construcción de palabras, de sílabas y de fonemas y, aunque los componentes musicales son diferentes de los del lenguaje, el proceso básico de aprendizaje puede ser similar.

\section{Método}

Con respecto al método de trabajo utilizado, este estudio, de corte cualitativo, se ha basado en el denominado análisis de información, entendido como la forma de investigar cuyo objetivo último es localizar, evaluar, seleccionar y sintetizar la información contenida en una serie de documentos (Dulzaides y Molina, 2004). Esta estrategia metodológica se imbrica con el análisis de contenido y el análisis documental, en los que la descripción y la interpretación de la información y de las ideas contenidas en los textos sometidos a estudio quedan configuradas como eje de la investigación llevada a cabo desde un enfoque sistemático y objetivo (Bardin, 2002).

En esta misma línea, López-Noguero (2002) y Solís (2003) señalan que la finalidad de este tipo de análisis es facilitar una aproximación cognitiva a los textos estudiados, en este caso a aquellos documentos que definen las relaciones existentes entre el aprendizaje de la música y el aprendizaje de la lengua materna, con el objetivo de recopilar su contenido y darlo a conocer a la comunidad de expertos.

Por último, Peña y Pirela (2007) afirman que el análisis documental es un excelente proceso mediador, una herramienta de peso teórico-metodológico para obtener la información contenida en un texto que responde a tres necesidades fundamentales: conocer lo que otros investigadores han hecho o están haciendo en un campo específico del conocimiento; conocer segmentos específicos de información de algún documento en particular; y conocer información relevante existente sobre un tema concreto.

\section{Las relaciones música-lengua materna en la pedagogía Willems}

El belga Edgar Willems (1890-1978) desarrolló su labor profesional e investigadora como pedagogo musical y maestro en Suiza. En sus teorías establece una estrecha relación entre psicología, sociología y música que le dan soporte a los paralelismos que construye entre el aprendizaje del lenguaje verbal y del lenguaje musical (Pascual, 2008). 
Su método parte de la idea de que tanto el aprendizaje de la lengua materna como el aprendizaje musical se basan en la escucha y en la imitación, por lo que el correcto desarrollo del oído es uno de los puntos esenciales de su metodología (Martí, 2016). Además, señala que el despertar de las habilidades musicales de los niños no pertenece al campo de la pedagogía musical, sino a su propia educación general; son las personas de su entorno familiar las que suelen desempeñar un papel fundamental en potenciar los aspectos auditivos y rítmicos de los más pequeños.

La madre puede atraer la atención del niño sobre los fenómenos sonoros y rítmicos y enseñarle las primeras melodías, a menudo en forma de canciones de cuna, rondas, etc. Ocurre que hay niños que pueden cantar buen número de canciones antes de la edad de dos años, aun antes de saber hablar. (Willems, 1979, p. 20)

La edad recomendada para comenzar a aprender música se sitúa entre los 4 y los 5 años (solo en los casos en los que los niños muestren capacidades musicales se podrá comenzar a los 3 años), y este aprendizaje se estructurará en cuatro grados, adecuados al momento psicológico de cada edad, pero "aplicables en sus principios a todas las edades y necesidades" (Fernández Ortiz, 2007, p. 50). Estos cuatro grados quedarían configurados de la siguiente manera:

- Grado 1. Iniciación musical articulada en cuatro grandes apartados: desarrollo auditivo y vocal, ejercicios rítmicos, canciones, movimiento corporal.

- Grado 2. Iniciación musical: transcripción de los primeros elementos musicales auditivos y rítmicos. Estimulación de la memoria y de la conciencia relativa.

- Grado 3. Iniciación de presolfeo y preinstrumento: se ordenan los fenómenos vividos, paso de lo concreto a lo abstracto, lateralización.

- Grado 4. Solfeo vivo y educación instrumental: lectoescritura rítmica y melódica, improvisación, organizaciones modaly tonal, tocar de oído, tocar a partir de la lectura, tocar de memoria improvisación instrumental.

Además, como señalan Willems y Chapuis (1989, p. 384), su método parte de unas bases filosóficas y psicológicas que establecen:

1. La unión entre la música, el ser humano y el cosmos.

2. Ordenamientos naturales y jerárquicos.

3. Un trabajo basado en la naturaleza íntima de los elementos constitutivos de la música, y no solamente en sus aspectos exteriores.

4. Un orden de desarrollo parecido al de la adquisición de la lengua materna.

La consecución efectiva de estos pasos está relacionada con el desarrollo sensorial, afectivo y mental de la persona, cuyas relaciones con el desarrollo musical se entienden como una ordenación natural en la que, como señalan Willems (1992) y Morales (2017), se dan las siguientes relaciones:

- Ritmo/vida física/instinto.

- Melodía/vida afectiva/afectividad.

- Armonía/vida mental/intelecto.

La música, como lenguaje que es, al igual que la lengua materna, precisa de una impregnación anterior a la práctica. Esta impregnación, como señala Fernández-Ortiz (2007), se consigue mediante la escucha (desarrollo sensorial), que implica un importante proceso de retentiva y diferentes intentos de reproducción de dicho lenguaje (desarrollo afectivo) llegando, finalmente, a la conciencia a través de la imitación (desarrollo mental).

Para que este proceso se pueda desarrollar plenamente, Willems (1979) entiende que es necesario centrar los esfuerzos en tres estadios fundamentales, musical, humano y social, de los que se derivan unos objetivos concretos e imprescindibles que facilitarán el aprendizaje musical:

- Musicales:

- Amar la música como lenguaje, arte y ciencia. 
Universidad Pedagógica Nacional

Facultad de Humanidades

- Cultivar la apertura al lenguaje y arte musicales.

- Desarrollar la sensibilidad auditiva y el sentido del ritmo.

- Desarrollar cualidades para el canto, el solfeo, la práctica musical y la armonía.

- Humanos:

- Desarrollar la memoria, la imaginación y la expresión.
- Desarrollar las facultades sensoriomotoras, afectivas, mentales e intuitivas.

- Sociales:

- Dirigirse a todos por igual, independientemente de sus capacidades iniciales, su edad y su origen.

- Trabajar en pequeños grupos siempre que sea necesario.

- Fomentar la música en familia.

Tabla 1. Relaciones entre el aprendizaje de la lengua materna y la adquisición del lenguaje musical consciente y estadios en los que se desarrollan

\begin{tabular}{|c|c|}
\hline Lenguaje & Música \\
\hline \multicolumn{2}{|c|}{ Orden psicológico de desarrollo. Actividad sensorial } \\
\hline Escuchar las voces & Escuchar los sonidos \\
\hline Mirar la boca del que habla & Mirar las fuentes sonoras, instrumentales o vocales \\
\hline \multicolumn{2}{|c|}{ Memoria, imaginación retentiva sensorial } \\
\hline Retener sin precisión elementos del lenguaje & Retener sonidos \\
\hline Retener sílabas, luego palabras & Retener sucesiones de sonidos y fragmentos de $\mathrm{m}$ \\
\hline
\end{tabular}

\section{Actividad afectiva. Imaginación retentiva afectiva}

Sentir el valor afectivo y expresivo del lenguaje

Volverse sensible al encanto de los sonidos, de las melodías

Actividad afectiva. Imaginación retentiva reproductora

Reproducir palabras aun sin comprenderlas

Reproducir sonidos, ritmos, pequeñas canciones

\section{Actividad mental. Imaginación retentiva mental}

Comprender el significado semántico de las palabras

Comprender el sentido de elementos musicales

Actividad mental. Imaginación reproductora, improvisación

Hablar uno mismo, inteligiblemente

Inventar ritmos, sucesiones melódicas

\section{Actividad mental. Conciencia mental reflexiva}

Aprender las letras, escribirlas y leerlas

Escribir al dictado
Aprender los nombres de las notas, escribirlas y leerlas

Escribir al dictado

Actividad inventiva. Imaginación constructiva con elementos conocidos

Hacer pequeñas redacciones y poemas Inventar melodías, pequeñas canciones

Actividad creadora. Imaginación creadora

Llegar a ser escritor, poeta o profesor Llegar a ser compositor, intérprete o profesor 
Teniendo en cuenta que música y lenguaje nacen de una necesidad de expresión y de comunicación, se observa en ambas disciplinas una evolución paralela que se irá cristalizando conforme se vayan cumpliendo las siguientes etapas: oír-escuchar-entender (Willems, 1964; Willems, 1992).

Desde esta perspectiva, la educación del oído se convierte en pilar de su método y la audición interior, generadora de capacidades mentales para improvisar y crear música, facilitará el camino hacia una interpretación comprendida, personal, sensible y fidedigna (Willems, 2001).

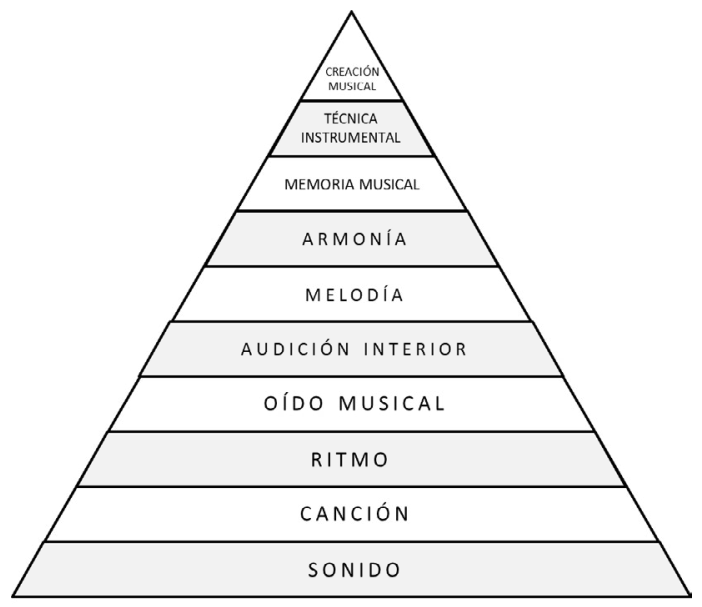

MÉTOdO WILLEMS-Elementos Musicales

Figura 1. Elementos musicales fundamentales de la metodología Willems

Fuente: elaboración propia según Martí (2016, p. 68).

Llevar a cabo su metodología supone una preparación específica del enseñante, que deberá tener conocimientos sobre los principios psicológicos de la educación y de la educación musical, y trabajar diferentes recursos en la educación sensorial del alumno (Martí, 2016).

\section{El método Suzuki y los paralelismos entre música y lengua materna}

El método conocido como la educación del talento fue creado y desarrollado por Shinichi Suzuki (1898-1998), violinista japonés que revolucionó el aprendizaje instrumental del momento. Este parte de la idea de que los niños están envueltos por los sonidos del idioma de su madre desde antes de nacer. Del mismo modo, si estuvieran rodeados de estímulos musicales podrían desarrollar unas capacidades musicales extraordinarias (Martí, 2016). El niño, por tanto, debe aprender el lenguaje musical igual que se aprende la lengua materna, a través de la escucha, la imitación y la aplicación de lo aprendido; esto le permitirá alcanzar las capacidades necesarias para llegar al proceso de lectura y escritura musical (Morales, 2017).

Suzuki (2004) parte del convencimiento de que cualquier niño es capaz de desarrollar habilidades superiores si se utilizan métodos adecuados en su entrenamiento. Esto se ve reflejado en el hecho de que todos ellos, independientemente de sus resultados académicos, hablan su lengua materna con fluidez y, prácticamente, sin esfuerzo, demostrando una gran habilidad para expresarse. A partir de esta reflexión, comienza a analizar detenidamente el proceso de aprendizaje de la lengua materna, y se da cuenta de que cuando un niño aprende una lengua, se siguen unas pautas muy bien definidas:

- El niño se va acostumbrando a los sonidos del lenguaje que escucha en el entorno que le rodea.

- Los padres, mediante la repetición constante, intentan que aprenda palabras.

- Cuando el niño comienza a hablar, los padres reaccionan muy positivamente, valorando todo avance por pequeño que parezca.

- A medida que el niño va hablando más y en más ocasiones, va mejorando sus construcciones lingüísticas y va perfeccionando su pronunciación.

Estas pautas, comunes en el aprendizaje primario de cualquier persona, quedan concretadas en los siguientes principios:

- Iniciación temprana: durante los primeros años de vida.

- El rol de los padres: los padres se convierten en profesores. 
- Audición: mediante la audición el niño conoce lo que tiene que aprender.

- Imitación: como uno de los sistemas más antiguos de aprendizaje.

- Repetición: con ella se interioriza sensorialmente aquello que se está aprendiendo.

- Sistema progresivo y secuenciado de aprendizaje, ya sea individual o en grupo.

En el desarrollo del lenguaje, el niño recibe estímulos, escucha y dice palabras en repetidas ocasiones. Igualmente, si un niño escucha y toca el repertorio musical repetitivamente, aprenderá la técnica y la música en pasos pequeños y secuenciales (Asociación Suzuki Internacional, s. f.).

En base a esto, el propio Suzuki (2004) afirmaba que, si los métodos educativos de la lengua materna se utilizaran en el aprendizaje de la música, las capacidades musicales del niño tendrían un desarrollo similar a las que se dan en la adquisición del lenguaje, y señalaba como puntos sustanciales de su método los siguientes axiomas:

1. Repetición constante.

2. Escucha de grabaciones.

3. Comentario positivo del trabajo del alumno.

4. Presentación continua de recitales.

5. Construcción de un repertorio.

6. Cada pieza es más fácil de memorizar.

7. Primero se desarrolla el oído y la intuición musical del alumno, después la lectura de las notas.

Para Bossuat (2007), los principios pedagógicos de Shinichi Suzuki tienen una correspondencia directa y fundamental con la existencia de un periodo prolongado de escucha, con la actitud positiva de las personas del entorno más cercano del niño y, finalmente, con la práctica cotidiana.



Figura 2. Principios básicos del método Suzuki

Fuente: elaboración propia según Martí (2016, p. 114).

Sobre la relación entre educación musical y lengua materna en la metodología Suzuki, Bossuat (2007), de nuevo, argumenta:

Indagó los mecanismos de adquisición de la lengua materna y su paralelismo con el estudio de un instrumento. Fascinado con la facilidad con la que aprenden los niños la lengua, profundizó en los motivos y condiciones que les permiten tal desenvoltura, y se planteó la siguiente cuestión: si este sistema funciona tan bien en el lenguaje, ¿por qué no se aplica a otros ámbitos como la música o las matemáticas?

Observó entonces que la adquisición de la lengua requiere un periodo prolongado de escucha, que prosigue incluso cuando el niño comienza a hablar. Se percató de que la actitud positiva de los seres cercanos es un estímulo primordial para los niños en el desarrollo lingüístico. Por otro lado, la práctica cotidiana de la lengua es, asimismo, una de las razones esenciales que nos permiten hablar con fluidez. De la lengua materna a la música no hay más que un paso, pues en ambos casos nos hallamos en el mundo de los sonidos. (p. 82) 
Uno de los puntos clave de esta pedagogía se fundamenta en la idea de que el aprendizaje de la lengua materna es el método educativo perfecto para el niño y, partiendo de ahí, la aplicación de este a la hora de enseñar otras facultades nos mostraría unos resultados que sobrepasarían a los obtenidos por los métodos vigentes (Suzuki, 2004).

Las características principales del proceso de desarrollo musical a través de este método son:

- Escuchar: el niño aprende palabras después de escucharlas una y otra vez. Escuchar música todos los días es fundamental, especialmente las piezas del repertorio a trabajar, para que las conozca con profundidad.

- Repetir: la repetición constante es imprescindible. En este método, el aprendizaje es acumulativo, como lo es el aprendizaje de vocabulario en el aprendizaje de la lengua materna.

- Alentar: el esfuerzo del niño requiere una recompensa en forma de elogio, sea cual sea su velocidad de aprendizaje.

- Repertorio graduado: los ejercicios técnicos son sustituidos por un repertorio definido. Cada pieza contiene el contenido técnico y musical necesario para el desarrollo interpretativo del niño de manera progresiva.

- Lectura postergada: al igual que el niño aprende a leer después de haber desarrollado la habilidad de hablar, es importante que la lectura musical se trabaje una vez desarrollada la técnica musical básica.

Aunque, en un principio, la filosofía Suzuki se centró en el desarrollo de habilidades y técnicas relacionadas con el aprendizaje del violín y del resto de instrumentos de cuerda frotada (viola, violonchelo y contrabajo), hoy, esta pedagogía se ha extendido al aprendizaje de cualquier instrumento musical. La estructura docente es similar para todos ellos, y consta de dos clases semanales: una individual, centrada en la adquisición de destrezas técnicas e interpretativas; y una colectiva, en la que se comparte lo aprendido individualmente, se refuerzan y perfeccionan los conocimientos adquiridos con la repetición constante y se desarrollan las habilidades de tocar en grupo (Suzuki, 2004).

En la actualidad, el método Suzuki se conoce y se pone en práctica en más de cincuenta países, y numerosos equipos de formadores especializados se encargan de mantenerlo vivo aplicándolo en conservatorios y escuelas de música. Además, ha traspasado las fronteras de la música denominada clásica extendiendo sus principios al estudio y aprendizaje de otros estilos como el jazz, la música étnica o la tradicional, entre otros.

\section{Las pedagogias Willems y Suzuki y las teorías sobre el desarrollo del lenguaje}

El tema que nos ocupa nos obliga a adentrarnos, al menos superficialmente, en algunas de las teorías más significativas relacionadas con el desarrollo evolutivo del niño. Si bien profundizar en el contenido de estas se desvía de los objetivos de este estudio, es importante tener en cuenta que un buen número de expertos (Bandura, 1987; Wallon, 2000; Piaget, 2008; Vygotsky, 2010; entre otros) ponen especial atención en los siguientes aspectos como elementos clave del desarrollo evolutivo del ser humano:

- La importancia del aprendizaje social.

- El papel fundamental de la observación y de la imitación.

- La interacción social del niño con adultos significativos para él.

- La construcción de su realidad conforme se va relacionando con el medio. 
Universidad Pedagógica Nacional

Facultad de Humanidades

Tabla 2. Estudio comparativo entre las etapas del desarrollo de Piaget y el desarrollo musical

\begin{tabular}{|c|c|c|}
\hline Etapa & Teoria del desarrollo de Piaget & Desarrollo musical \\
\hline \multirow{9}{*}{ 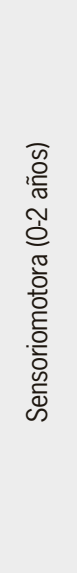 } & Manipulación de objetos para descubrir su utilidad & Diferenciación entre intensidad, altura, timbre, duración o textura \\
\hline & Gran importancia del juego & Sensación placentera al escuchar sonidos \\
\hline & Relación de acciones que anteriormente eran aisladas & Reflejos dinámicos y capacidad para matizarlos \\
\hline & Confusión entre realidad y deseo & Asimilación de esquemas sonoros \\
\hline & & Capacidades perceptivas básicas para localizar y discriminar sonidos \\
\hline & & Emisión de vocales y consonantes \\
\hline & & Expresión musical mediante canciones \\
\hline & & Improvisación en canciones repetitivas \\
\hline & & Distinción entre música y ruido \\
\hline \multirow{11}{*}{ 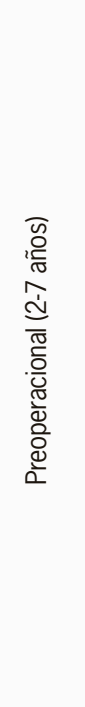 } & Interacción con el entorno & Imitación, comunicación, interpretación y creación básicas \\
\hline & Curiosidad sobre lo percibido & Capacidad para representar gráficmente estímulos sonoros \\
\hline & Egocentrismo y antropocentrismo & Reproducción de canciones completas \\
\hline & Desarrollo de la motricidad gruesa & Capacidad para seguir la pulsación de una canción \\
\hline & Mayor equilibrio y sincronización & Aumento de la capacidad de entonación \\
\hline & & Capacidad para seguir el ritmo con extremidades superiores \\
\hline & & Distinción entre pulso y acento \\
\hline & & Invención de ritmos y melodías \\
\hline & & Introducción a los instrumentos de percusión \\
\hline & & Percepción armónica \\
\hline & & Desarrollo progresivo de la voz \\
\hline \multirow{6}{*}{ 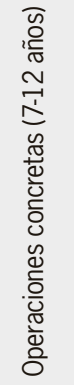 } & Disminución del pensmiento egocentrista & Capacidades fonadoras muy desarrolladas \\
\hline & Capacidad para agrupar & Desarrollo de la expresión corporal \\
\hline & Discriminación de los diferentes aspectos de un estímulo & Capacidades polirrítmicas y polifónicas \\
\hline & Desarrollo de la motricidad fina & Crítica e identificación de sus propias creaciones musicales \\
\hline & Conciencia del pensamiento propio & Comienzo del cambio de voz \\
\hline & Mayor facilidad para resolver problemas & \\
\hline \multirow{6}{*}{ 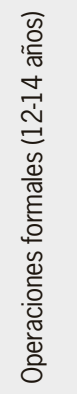 } & Visión más abstracta del mundo & Expresión instrumental más controlada y de mayor calidad \\
\hline & Uso de la lógica formal & Trabajo vocal más complicado \\
\hline & Reversibilidad & Expresión corporal más controlada y de mayor calidad \\
\hline & Idea de causa y efecto & Capacidad para pensar en una futura especialización musical \\
\hline & Formulación de hipótesis y búsqueda de soluciones a problemas & \\
\hline & Razonamiento en contra de hechos & \\
\hline
\end{tabular}

Fuente: elaboración propia según Alsina (1997, p. 31) y Swanwick (2006, pp. 84-90). 
En relación con los estudios y teorías relacionadas con el desarrollo del lenguaje, Seco (1993), Navarro (2003); Fernández, González, Martínez y Pampín (2010); Fernández-Pérez (2015); entre otros, establecen los siguientes criterios básicos para que el niño desarrolle el habla de forma correcta:

- Su maduración neurológica.

- La estimulación familiar y del entorno recibida.

- La interacción con los adultos de su entorno.

- Las propias experiencias del niño con su entorno.

- Las diferencias individuales de cada niño.

- La repetición, la imitación y los impulsos creativos.

En esta misma línea, Fernández-Pérez (2015) resalta la importancia de los estímulos y de los elementos del entorno del niño desde tres ámbitos importantes: desde el plano psicológico-cognitivo, ya que se trabaja la atención, la motivación y la concentración; desde el psicológico-emocional, que permitirá controlar las emociones para un rendimiento sin conflictos; por último, desde el plano de la socialización, con el que se incentivarán intercambios, prácticas de interacción y reconocimiento de los otros.

Estos principios, como recoge el Instituto Nacional de Tecnologías Educativas y de Formación del Profesorado del Gobierno de España (INTEF, s. f.) se ven reflejados en las teorías de la adquisición del lenguaje de Skinner, Chomsky, Vygotsky y Bruner, a saber:

- Skinner se centra en la importancia de la estimulación y del refuerzo positivo focalizado en la lengua materna y en la imitación.

- Chomsky antepone las capacidades innatas del niño como pilar en el desarrollo del lenguaje.

- Vygotsky, por su parte, afirma el ámbito social dentro del contexto cultural del niño marcará su aprendizaje.

- Finalmente, Bruner señala que el lenguaje se aprende a través de la interacción del niño con la madre.
Por otro lado, Navarro (2003) recoge las teorías de Jacobson, Alarcos y Piaget y nos recuerda la importancia de la calidad de las relaciones entre el niño y el adulto como principio básico de la comunicación y de la adquisición del lenguaje y señala dos etapas fundamentales en este proceso:

- Etapa prelingüística (de 0 a 12 meses): emisión de los primeros sonidos, realización de gestos relevantes, reacción a la lengua y a acciones que percibe. Aparición de formas básicas de comunicación con su entorno.

- De 0 a 3 meses: emisión de sonidos indeterminados.

- Entre 3 y 4 meses: emisión de sonidos correspondientes a sílabas concretas.

- De 6 a 12 meses: balbuceos imitativos y aparición de patrones de entonación.

- A partir de los 10 meses, comienza a adjudicar palabras a objetos.

- Etapa lingüística (a partir de los 12 meses): uso del lenguaje con intenciones comunicativas, adquisición de sus elementos y la forma de combinarlos con significado y con una función.

- De 12 a 24 meses: comienza a decir palabras sueltas.

- De 2 a 4 años: construye frases cortas y crece el número de palabras que conoce y usa.

- A los 4 años domina construcciones sintácticas simples.

- De los 4 a los 7 años: dominio de la pronunciación.

De lo anterior se desprende que existen paralelismos claros entre el aprendizaje musical propuesto por Willems y Suzuki y el aprendizaje y desarrollo del lenguaje en el niño. Así, el aprendizaje social y en grupo, la observación y la imitación, la interacción niño-adulto, la estimulación tanto familiar como del entorno y la repetición son elementos fundamentales comunes a ambos procedimientos. 
Universidad Pedagógica Nacional

Facultad de Humanidades

Tabla 3. Paralelismos y divergencias entre los procesamientos musical y lingüístico

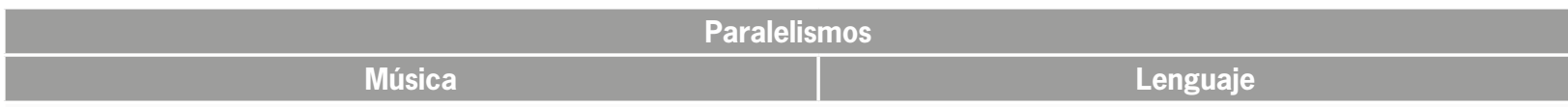

Actividades sociales o grupales con las que se constituye, se comunica y se comparten pautas de comportamiento y estados mentales y emocionales entre miembros de una comunidad

El tiempo es una condición básica para conformarse como tal

Actividades reglamentadas que también pueden ser individuales

Consideración artística

Comparten formas básicas de expresión (características acústicas, tonalidad, sonoridad, timbre, tiempo, ritmo)

Necesitan productor y receptor para darse en su máxima expresión

Rasgos acústicos y temporales equivalentes

Capacidad de generar infinitas combinaciones a partir de un inventario finito de elementos

Representación icónica de objetos y eventos

Evocación y transmisión de sentimientos y emociones

\begin{tabular}{|l|l|}
\hline \multicolumn{1}{c}{ Música } & \multicolumn{1}{c}{ Divergencias } \\
\hline Constituida solo por fonemas & Constituida por monemas y fonemas \\
\hline No está claro que sea un lenguaje & Sí es un lenguaje \\
\hline No parece ofrecer ventajas adaptativas tan obvias & Permite comunicar mensajes complejos, sirve para controlar \\
como las del lenguaje & y organizar el pensamiento y para planificar acciones \\
\hline La sintaxis musical representa relaciones armónicas & La sintaxis lingüística refleja relaciones semánticas \\
\hline No puede expresar actitudes proposicionales & Expresión de actitudes proposicionales \\
\hline Expresa emociones y sentimientos, pero no puede representarlos & Expresa y representa emociones y sentimientos \\
\hline Su significado no es composicional & Significado descomponible en unidades discretas \\
\hline
\end{tabular}

Fuente: elaboración propia según lgoa (2010) y Eisner (2013).

Por otro lado, es importante tener en cuenta algunas divergencias (tabla 3), especialmente las relacionadas con las especificidades propias de cada uno de los sistemas significantes, lo que los configura como facultades con entidad propia (Igoa, 2010). Sin embargo, las actividades que desarrollan habilidades lingüísticas y musicales conjuntamente presentan numerosos e importantes rasgos comunes en las dimensiones del sonido, la sintaxis y el significado, confirmando la pertinencia de las teorías sostenidas y desarrolladas por ambos pedagogos musicales.

\section{Las relaciones música-lenguaje desde la neurociencia}

Estudios recientes indican que tanto hacer como escuchar música requiere de la actividad coordinada de diferentes módulos cerebrales, lo que provoca una comunicación interhemisférica y, por tanto, un uso de los pensamientos convergente y divergente. Esto significa que la música se distribuye por todo el cerebro, en contra de la antigua idea de que el arte y la música se procesan en el hemisferio derecho, mientras que el lenguaje y las matemáticas lo hacen en el izquierdo. Además, Levitin (2008) afirma que oír, componer e interpretar música requieren la intervención de casi todas las áreas cerebrales, y exige la participación de casi todo el subsistema neurológico.

Esto pone de manifiesto, entre otros asuntos, que las actividades musicales utilizan el cerebro de forma globalizada (Peñalba, 2016), y que la música posee la capacidad de secuestrar sistemas cerebrales construidos para otros fines, tales como el lenguaje, la emoción y el movimiento (Manes, 2015). En esta línea, y centrándonos en las relaciones neuronales entre música y lenguaje, Koelsch (2011) da un paso más: "el cerebro humano, sobre todo en edades tempranas, no trata la música y el lenguaje como 
dominios separados, sino que trata al lenguaje como un caso especial de música” (p. 6).

Sobre esto, Díaz (2010) señala que distintos instrumentos de medición y análisis de la actividad cerebral como la neuroimagen, el electroencefalograma y la resonancia magnética funcional han mostrado que en los estímulos auditivos, además de activarse ambas regiones auditivas primarias, se activan el polo temporal izquierdo, la circunvolución frontal inferior y el área frontopolar, sugiriendo que se necesitan procesamientos lingüísticos y cognitivos en la respuesta general a la música, entrando en juego los estratos neocorticales que se entrelazan con los sistemas cerebrales del lenguaje.

Esto nos indica, por consiguiente, que la participación de amplias zonas del hemisferio izquierdo durante la experiencia musical se debe a las propiedades gramaticales y simbólicas de la música que, como ocurre en el lenguaje proposicional, activan los sistemas representacionales y simbólicos del sistema mente/cerebro. Esto nos da a entender que las reglas musicales, como en el lenguaje verbal, siguen unas pautas sintácticas o, al menos, ofrecen ciertas expectativas sintácticas (Díaz, 2010).

Profundizando más en las áreas cerebrales comunes en la percepción de la música y el lenguaje, García, Del Olmo y Gutiérrez (2014) concretan que existe una respuesta similar de las regiones 41 y 42 de la corteza auditiva primaria en la escucha musical y el habla, que se da una activación de la región 22 de la corteza auditiva secundaria tanto en la escucha y la comprensión de la palabra como en la audición de escalas, la recreación interna de sonidos o la representación melódica, que la participación del área 40 (giro supramarginal) se produce en la comprensión del símbolo lingüístico y en la lectura musical y, finalmente, que el área de Broca reacciona en la actividad motora relacionada con el habla, así como en la ejecución rítmica y musical.

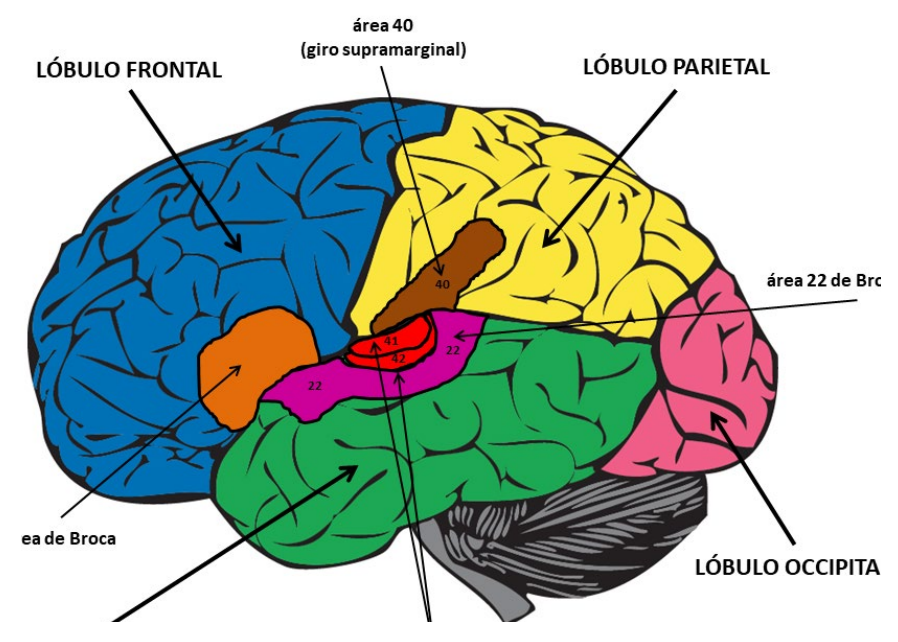

Figura 3. Lóbulos cerebrales y áreas comunes en la percepción de la música y el lenguaje

Fuente: elaboración propia.

De lo anterior se puede deducir que, aunque los dominios lingüístico y musical ocupan distintas áreas cerebrales, usan los mismos recursos para activar e integrar las representaciones sintácticas y, por tanto, disponen de un circuito neuronal común.
De este modo, música y lenguaje comparten procesos neuronales de integración de eventos secuenciados, que median decisivamente en el aprendizaje de ambos sistemas significantes (García, Del Olmo y Gutiérrez, 2014). 
En consecuencia, esta mirada desde las neurociencias pone en valor, de nuevo, las aportaciones teórico-musicales de Willems y Suzuki en las que establecen una serie de analogías en el desarrollo de habilidades musicales y lingüísticas de los niños, otorgando un mayor peso específico a sus métodos de aprendizaje musical.

\section{Conclusiones}

Con este estudio se han intentado presentar, analizar y justificar aquellas investigaciones que indican que el lenguaje y la música comparten una red de procesamiento sintáctico común que abre la posibilidad a la existencia de representaciones paralelas en ambos dominios, el musical y el lingüístico. En este sentido, se han ofrecido diferentes aportaciones que analizan los paralelismos y las divergencias existentes entre el aprendizaje musical y el aprendizaje del lenguaje en niños en edades tempranas, tanto desde las teorías del desarrollo lingüístico como desde la neurociencia y la pedagogía musical. Sin embargo, dichas divergencias, relacionadas con los ámbitos más específicos de cada uno de estos dominios, no muestran unos valores decisorios que pudieran contradecir o descartar las coincidencias encontradas en las investigaciones más recientes sobre estas representaciones paralelas y sus implicaciones.

Dos de las metodologías musicales, pioneras en el tema que nos ocupa, que abrieron las puertas a estudios posteriores desde diferentes ámbitos disciplinares y se encargaron de recoger, analizar y relacionar los procesos de aprendizaje de la música y de la lengua buscando conexiones interdisciplinares, son las desarrolladas por Edgar Willems y Shinichi Suzuki. Ambos pedagogos basaron sus teorías en las similitudes existentes en el desarrollo de capacidades lingüísticas y musicales en los primeros años de vida del niño, encontrando puntos coincidentes con las teorías del desarrollo evolutivo del ser humano, con las de la adquisición del lenguaje y con las neurociencias encargadas de estudiar las funciones cognitivas que se producen en los procesos musical y lingüístico.
En relación a las teorías del desarrollo evolutivo y de la adquisición del lenguaje, la observación, la imitación, el aprendizaje en grupo, el refuerzo positivo, la repetición bien enfocada, la interacción niño/ adulto y la estimulación familiar y del entorno más cercano del niño, son aspectos fundamentales que recogen los dos ámbitos disciplinares como criterios básicos en el buen desarrollo evolutivo y lingüístico del ser humano, y estos son compartidos con los métodos Willems y Suzuki, en los que quedan definidos como principios básicos del aprendizaje musical.

Por su parte, el acercamiento a las investigaciones llevadas a cabo por la neurociencia nos ha permitido conocer más a fondo las conexiones cerebrales ante los procesamientos lingüístico y musical y las coincidencias en la activación de diferentes áreas o regiones hemisféricas, comprobando que existe un circuito neuronal común entre los dominios musical y del lenguaje.

Esto nos ha permitido comprobar cómo las pedagogías Willems y Suzuki mantienen una fuerte correlación con estudios posteriores desde diferentes áreas de conocimiento, aportando a ambas un alto grado de confiabilidad y proporcionándoles un importante peso específico dentro de las metodologías musicales desarrolladas en el siglo xx.

Para terminar, hay que destacar que tanto la pedagogía Willems como la pedagogía Suzuki se han asentado fuertemente en un buen número de países alrededor del mundo. Las diferentes asociaciones y escuelas de enseñanza musical vinculadas a estas metodologías centran una parte importante de su labor en la formación del profesorado y en la organización de jornadas y congresos en los que presentan sus líneas de trabajo actuales y los avances alcanzados en el desarrollo del lenguaje musical a imagen del desarrollo de la lengua materna. 


\section{Referencias}

Adorno, T. (1997). Fragmento sobre las relaciones entre música y lenguaje. Revista Colombiana de Psicología, 5-6, 174-177. https://dialnet.unirioja.es/servlet/ articulo? codigo $=4895321$

Adorno, T. (2000). Sobre la música. Paidós.

Álamos, J. E. y Pérez, M. (2015). Paralelos cognitivos entre música y lengua materna en estudiantes de educación primaria. Un estudio sobre la influencia del lenguaje verbal y de las características melódicas específicas en la memoria musical a corto plazo. Revista Electrónica de LEEME, 35, 1-27. https://dialnet.unirioja.es/ servlet/articulo? codigo $=5385581$

Alonso, M. F., Suárez, W., Espinoza, M. J. y Ramírez, P. (2016). Estimulación auditiva como herramienta terapéutica en el trastorno del lenguaje infantil. Medicina Naturista, 1(10), 21-27. https://dialnet. unirioja.es/servlet/articulo?codigo $=5302178$

Alsina, P. (1997). El área de educación musical. Propuestas para aplicar en el aula. Barcelona: Graó.

Asociación Suzuki Internacional (s. f.). Método Suzuki y su filosofía. Asociaciones Suzuki de los Países de América Latina, 2, 34-51. http://catarina.udlap. $\mathrm{mx} / \mathrm{u}$ _dl_a/tales/documentos/lmu/duran_f_md/ capitulo2.pdf

Bandura, A. (1987). Pensamiento y acción. Fundamentos sociales. Martínez Roca.

Bardin, L. (2002). El análisis de contenido. Akal.

Bossuat, C. (2007). Shinichi Suzuki. En M. Díaz y A. Giráldez (coords.), Aportaciones teóricas y metodológicas a la educación musical (pp. 79-86). Graó.

Díaz, J. L. (2010). Música, lenguaje y emoción: una aproximación cerebral. Salud Mental, 6(33), 543-551. http://www.scielo.org.mx/scielo.php?script=sci_arttext\&pid=S0185-33252010000600009

Dulzaides, M. E. y Molina, A. M. (2004). Análisis documental y de información: dos componentes de un mismo proceso. Revista Cubana de Información en Ciencias de la Salud, 2(12). http://scielo.sld.cu/pdf/ aci/v12n2/aci11204.pdf

Eisner, G. (2013). ¿La música más acá o las palabras más allá? El controversial vínculo entre música y lenguaje verbal. Neuma. Revista de Música y Docencia Musical, 6(1), 30-46. https://dialnet.unirioja.es/servlet/ articulo? codigo $=4713122$
Fernández-Ortiz, J. (2007). Edgar Willems. En M. Díaz y A. Giráldez (coords.), Aportaciones teóricas y metodológicas a la educación musical. Una selección de autores relevantes (pp. 43-53). Graó.

Fernández-Pérez, M. (2015). Lenguaje infantil y medidas de desarrollo verbal. Ensayos. Revista de la Facultad de Educación de Albacete, 30(2), 53-69. https:// dialnet.unirioja.es/servlet/articulo?codigo $=5386465$

Fernández, S., González, A. I., Martínez, M. E. y Pampín, L. (2010). Lectura y escritura: introducción al desarrollo del lenguaje. Revista Padres y Maestros, 333, 1-4. http://revistas.upcomillas.es/index.php/ padresymaestros/article/view/1242/1056

Fubini, E. (2004). Música y lenguaje en la estética contemporánea. Alianza.

García, E., Del Olmo, M. J. y Gutiérrez, E. (2014). Educación musical y desarrollo cognitivo asociado. Música y Educación. Revista Internacional de Pedagogía Musical, 97, 28-41.

Herrera, L. y Lorenzo, O. (2006). Música como lenguaje y lengua materna. Música y Educación, 66, 87-102.

Igoa, J. M. (2010). Sobre las relaciones entre la música y lenguaje. Epistemus, 1(1), 97-125. https://revistas. unlp.edu.ar/Epistemus/article/view/2703

Instituto Nacional de Tecnologías Educativas y de Formación del Profesorado (INTEF) (s. f.). Principales teorías de la adquisición del lenguaje. Madrid: Formación en Red del INTEF. http://formacion.intef. es/pluginfile.php/154123/mod_imscp/content/1/ principales_teoras_en_la_adquisicin_del_lenguaje. html

Koelsch, S. (2011). Towards a neural basis of processing musical semantics. Physics of Life Reviews, 8(2), 89-105. https://doi.org/10.1016/j.plrev.2011.04.004

Levitin, D. J. (2008). Tu cerebro y la música. El estudio científico de una obsesión humana. Barcelona: RBA.

López-Noguero, F. (2002). El análisis de contenido como método de investigación. XXI. Revista de Educación, 4, 167-179. INTEF http://rabida.uhu.es/dspace/bitstream/handle/10272/1912/b15150434.pdf

Manes, F. (14 de septiembre de 2015).¿Quéle hacela música a nuestro cerebro? ElPaís.com https://elpais.com/ elpais/2015/08/31/ciencia/1441020979_017115.html

Martí, J. M. (2016). Aprendizaje musical para niños. Metodologías y sistemas pedagógicos de la didáctica musical. Redbook Ediciones, Ma Non Troppo-Taller de Música. 
Morales, A. (2017). Fundamentos psicopedagógicos y principios de la educación musical en primaria. En R. Cremades (coord.), Didáctica de la educación musical en primaria (pp. 1-18). Paraninfo Universidad.

Navarro, M. (2003). Adquisición del lenguaje. El principio de la comunicación. Cauce. Revista de Filología y su Didáctica, 26, 321-347. https://dialnet.unirioja.es/ servlet/articulo?codigo $=1086886$

Palacios, J. I. (2005). La universidad y la investigación musical: de la teoría a la praxis. Revista Interuniversitaria de Formación del Profesorado, 19(1), 123-156.

Pascual, P. (2008). Didáctica de la música. PearsonPrentice Hall.

Peña, T. y Pirela, J. (2007). La complejidad del análisis documental. Información, Cultura y Sociedad, 16, 55-81. https://dialnet.unirioja.es/servlet/ articulo? codigo $=2501044$

Peñalba, A. (2017). La defensa de la educación musical desde la neurociencia. RECIEM. Revista Electrónica Complutense de Investigación en Educación Musical, 14, 109-127. https://doi.org/10.5209/RECIEM.54814

Piaget, J. (2008). Psicología del niño. Morata.

Saffran, J. R., Johnson, E. K., Aslin, R. N. y Newport, E. I. (1999). Statistical learning of tono sequences by human infants and adults. Cognition, 70, 27-52.

Seco, E. (1993). El lenguaje en las edades tempranas. Revista Interuniversitaria de Formación del Profesorado, 18, 29-36. https://dialnet.unirioja.es/ servlet/articulo? codigo $=117785$
Solís, I. A. (2003). El análisis documental como eslabón para la recuperación de información y los servicios. Monografías.com. https://www.monografias.com/ trabajos14/analisisdocum/analisisdocum.shtml

Suzuki, S. (2004). Educados con amor. El método clásico de la educación del talento. Alfred Music.

Swanwick, K. (2006). Música, pensamiento y educación. Ediciones Morata.

Vygotsky, L. (2010). Pensamiento y lenguaje. Paidós.

Wallon, H. (2000). La evolución psicológica del niño. Crítica.

Willems, E. (1964). El ritmo musical. Estudio psicológico. Eudeba.

Willems, E. (1979). Las bases psicológicas de la educación musical. Editorial Universitaria.

Willems, E. (1992). Nuevas ideas filosóficas sobre la música y sus aplicaciones prácticas. Folios. Revista de la Facultad de Artes y Humanidades, 3, 103-110. http://revistas.pedagogica.edu.co/index.php/RF/ article/view/5351

Willems, E. (2001). El oído musical. La preparación auditiva del niño. Paidós.

Willems, E. y Chapuis, J. (1989). Características del método Willems de Educación Musical. Principios válidos tanto para la iniciación musical de los niños pequeños como para la preparación al solfeo, instrumento y creación musical. Música y Educación. Revista Trimestral de Pedagogía Musical, 2, 383-390. 